\title{
A Statistical Atlas of Prostate Cancer for Optimal Biopsy
}

\author{
Dinggang Shen ${ }^{1}$, Zhiqiang Lao ${ }^{1}$, Jianchao Zeng ${ }^{2}$, Edward H. Herskovits ${ }^{1}$, \\ Gabor Fichtinger ${ }^{3}$, Christos Davatzikos ${ }^{1,3}$ \\ ${ }^{1}$ Department of Radiology, Johns Hopkins University \\ ${ }^{2}$ ISIS Center, Radiology Department, Georgetown University Medical Center \\ ${ }^{3}$ Center for Computer-Integrated Surgical Systems and Technology, Johns Hopkins University \\ Email: dgshen@cbmv.jhu.edu, hristos@rad.jhu.edu
}

\begin{abstract}
This paper presents a methodology of creating a statistical atlas of spatial distribution of prostate cancer from a large patient cohort, and uses it for designing optimal needle biopsy strategies. In order to remove inter-individual morphological variability and determine the true variability in cancer position, an adaptive-focus deformable model (AFDM) is used to register and normalize prostate samples. Moreover, a probabilistic method is developed for designing optimal biopsy strategies that determine the locations and the number of needles by optimizing cancer detection probability. Various experiments demonstrate the performance of AFDM in registering prostate samples for construction of the statistical atlas, and also validate the predictive power of our atlas-based optimal biopsy strategies in detecting prostate cancer.
\end{abstract}

\section{Introduction}

Prostate cancer is the second leading cause of death for American men. Transrectal Ultrasonography-guided symmetric needle biopsy has been widely used as a gold standard for the diagnosis and staging of prostate cancer. However, biopsy is currently performed in a rather empirical way, since cancer is mostly undetectable in the routinely used ultrasound images. This results in a significant number of prostate cancer cases being undetected at their initial biopsy. For example, the systematic sextant biopsy protocol [1] is the most common biopsy protocol. However, some studies have shown that this protocol results in a positive predictive value of only around $30 \%$ [2]. Other clinical studies have suggested that the sextant technique may not be optimal and have investigated new biopsy protocols that might yield significantly better results $[3,4]$. Obviously, if the biopsy protocol can be optimized to increase the chances of detecting prostate cancer, according to some objective and quantitative criteria, then significant improvement in diagnostic accuracy should be expected.

Some researchers have investigated the possibility of using a large number of patient histopathological images to determine prostate regions that are most likely to develop cancer, and therefore should be sampled during biopsy [5]. Those techniques, however, are limited by inter-individual morphological variability, which reduces both the statistical power in detecting associations and the spatial specificity of these methods, which is often limited to relatively coarse subdivisions of the prostate. In this paper, we propose a methodology that overcomes both of these limitations. In order to reduce inter-individual variability, we use AFDM [6,7], which spatially normalize the prostate images to a canonical coordinate system with high accuracy. With accurate

W. Niessen and M. Viergever (Eds.): MICCAI 2001, LNCS 2208, pp. 416-424, 2001.

(C) Springer-Verlag Berlin Heidelberg 2001 
registration of the prostate images of a large number of patients, a statistical atlas of cancer distribution can be created and further applied in suggesting optimal biopsy strategies. As to the statistical analysis, we not only look at the probability of developing cancer at individual locations, but also develop a full statistical predictive model that takes into account the spatial correlation of cancer incidence between different prostate regions. Our rationale is that regions between which cancer incidence is very highly correlated need not be sampled simultaneously, as opposed to regions between which cancer occurrence is relatively independent. These models are used in an optimization framework for estimation of optimal needle biopsy strategies.

This paper is organized as follows. In Section 2, we build a methodology for deformable registration and normalization of prostate samples. In Section 3, we create a statistical atlas of the spatial distribution of prostate cancer and develop a probabilistic method for designing optimal biopsy strategies that best predict the presence of prostate cancer in a particular patient. In Section 4, we demonstrate the performance of AFDM in registering prostate samples, and validate the predictive power of our atlasbased optimal biopsy strategies in detecting clinically significant cancer in our existing prostate database.

\section{Spatial Registration and Normalization of Prostate Samples}

The major problem in developing a spatial normalization method is determining correspondences. We have developed a deformable shape modeling framework, for segmentation and reconstruction of anatomical shapes, and for determining morphologybased correspondence across individuals, from tomographic images [6,7]. This framework is based on our AFDM. In AFDM, for a given set of structures, a shape model is first constructed to represent a typical shape of these structures. This shape model includes two kinds of information: information about the geometry of the structures and information about the statistical variation of these structures within a given population. In the application stage, the deformable shape model is placed in an image with the structures of interest, and is subsequently let free to deform according to features extracted from the images, seeking objects that have similar geometry, but also objects that fall within the expected range of shape variation.

In this section, we use AFDM as a registration method to spatially normalize the external and internal structures of the prostate samples, such as the capsule and the urethra. In particular, we select one typical prostate as the template (Fig. 1a), and other subjects are warped into the space of this template. The warping is performed in two stages. First, AFDM is used to reconstruct the shape of each structure and to determine point correspondences between the subjects and the template. Second, these point correspondences are interpolated elsewhere in the space of the prostate by using an elastic warping technique [8]. Since AFDM is the cornerstone of our deformable registration method, it is particularly redesigned for prostate application next. 


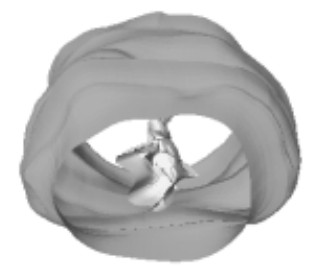

(a)

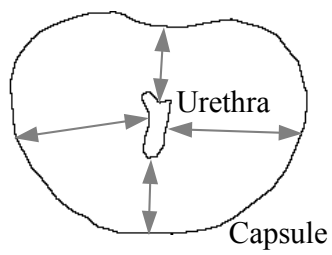

(b)

Fig. 1. A prostate surface model. (a) 3D display, and (b) a cross-section.

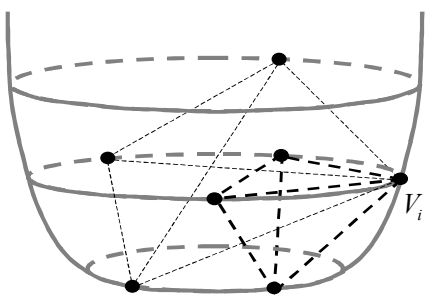

Fig. 2. Attributes defined as volumes of tetrahedra in $3 \mathrm{D}$.

\subsection{Spatial Connections between Distant Structures of the Prostate Surface Model}

AFDM comprises several inter-connected surfaces, each representing the anatomy of a structure of interest. Fig. 1a shows a 3D prostate model with two surfaces that represent the capsule and the urethral boundaries. In order to allow a deformation imposed on a segment of one surface (such as the capsule) to rapidly propagate to the segments of the other surface (such as the urethra) in a hierarchical fashion, connections between these two surfaces are added (gray arrows in Fig. 1b), effectively rendering distant surface patches as neighbors. Therefore, at a coarse scale, large segments are connected together, and thus deformations have a strong effect on the distant segments. At a finer scale, the deformations have a more local character.

\subsection{Affine-Invariant Attribute Vector on Each Model Point}

In order to capture the geometry of anatomical structures in a hierarchical fashion, we introduced the concept of an attribute vector that is attached to each point of the prostate surface model and reflects the geometric structure of the model from a global scale to a local scale. The attribute vectors are an important aspect of AFDM, since they provide a means for finding correspondences across individuals by examining the similarity of the underlying attribute vectors. In 3D case, each attribute is defined as the volume of a tetrahedron (see Fig. 2), formed by a model point $V_{i}$ and any three points in its certain neighborhood layer. For each model point $V_{i}$, the volumes that are calculated from different neighborhood layers of its surface segment can be stacked into an attribute vector $F\left(V_{i}\right)$, which can be made affine-invariant as $\hat{F}\left(V_{i}\right)$ by normalizing it over the whole model [6,7].

\subsection{Energy Definition of Prostate Surface Model}

Our deformable model is very robust to local minima, since the local energy term $E_{i}$ [7], that is composed of two terms $E_{i}^{\text {model }}$ and $E_{i}^{\text {data }}$, is defined on the surface segment of the model point $V_{i}$, rather than a single model point $V_{i}$. The model energy term $E_{i}^{\text {model }}$ is defined to allow AFDM determine correspondences, in addition to segmenting structures of interest. In particular, the model energy term $E_{i}^{\text {model }}$ is defined as the difference between the attribute vectors of the model and its deformed configuration, 
and it is given by $E_{i}^{\text {model }}=\left\|\hat{F}^{\text {Def }}\left(V_{i}\right)-\hat{F}^{\mathrm{Mdl}}\left(V_{i}\right)\right\|^{2}$, where $\hat{F}^{\text {Def }}\left(V_{i}\right)$ and $\hat{F}^{\mathrm{Mdl}}\left(V_{i}\right)$ are respectively the normalized attribute vectors of the deformed model configuration and the model at the point $V_{i}$. As to the data energy term, $E_{i}^{\text {data }}$, it is designed to move the deformable model towards an object boundary. Since our deformation mechanism deforms a surface segment around each model point $V_{i}$ at a time, we design a data energy term $E_{i}^{\text {data }}$ that reflects the fit of the whole surface segment, rather than a single model point, with image edges.

\subsection{Adaptive-Focus Deformation Strategy}

In our previous work we have determined that model adaptivity is very important for robust segmentation and correspondence estimation. Adaptivity was embedded in AFDM by employing an external force mechanism in a hierarchical way, starting with relatively easier to find structures and gradually shifting focus to other structures, as the model deforms into a configuration closer to the shape of an individual structure. We explore the utility of adaptive modeling further in the registration of prostate samples (see Fig. 3). Two types of prior knowledge about the prostate shapes are available. First, the attribute vectors of the model points along the two open surface boundaries of the prostate shape model are very distinct, compared to other attribute vectors of other model points, such as in the middle cross-section of the prostate model. With this prior knowledge, we will focus on the two open surface boundaries in the initial deformation stages, which will lead to a rough match between the prostate model and the subject. Second, the prostate capsule is more accurately and reliably outlined in the histopathological samples, compared to the urethra that does not include soft tissue and therefore is very deformed in the specimens. This prior knowledge suggests us to focus on the prostate capsule first, and then shift focus to the urethra, as more information about the urethral shape is gathered from the subject.

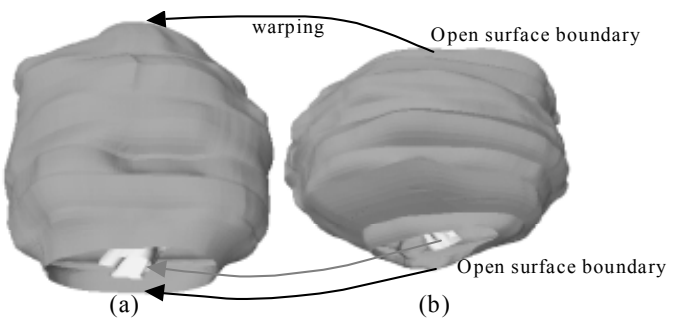

Fig. 3. The procedure of warping a prostate subject to the template. (a) Side view of the template given in Fig. 1, (b) Side view of the subject that is the same as the one in Fig. 4(b1).

\section{Optimal Needle Biopsy Strategy}

After warping all prostate subjects into the space of the template by AFDM, we construct the 3D statistical atlas of spatial distribution of prostate cancer, and we further design optimal needle biopsy strategies for diagnosing prostate cancer in a patient. In the next, we describe an algorithm to design optimal $K$-biopsy (biopsy with a number $K$ of needles) strategy by minimizing the probability of missing the cancer. 


\subsection{Optimization}

A $K$-biopsy strategy can be optimized by minimizing the probability that none of the $K$ needles detects cancer. This probability is defined by

$$
P\left(B\left(x_{i}\right)=\mathrm{NC}, i=1, . ., K\right),
$$

where $B\left(x_{i}\right)$ is the biopsy outcome at location $x_{i}$, and NC denotes a negative cancer detection result. Location $x_{i}$ can be anywhere inside of a $3 \mathrm{D}$ prostate template. We can find the configuration that minimizes this probability by using standard optimization methods. Since the probability in (1) is likely to have many local minima, we use the simulated annealing technique to find globally optimal values for $x_{1} \ldots x_{K}$. We start with an initial guess for the coordinates of the $K$ needles, and then we iteratively change these values toward a direction that decreases the probability function in (1). Initially, changes in the direction that increases the probability in (1) are allowed, but these steps are progressively discouraged more and more as the algorithm proceeds, as customary in random optimization methods.

\subsection{A Fast Heuristic Method}

The search space in our optimization problem is very large, since each of the $K$ needle coordinates can be any voxel within the prostate template. Therefore, in order to make the simulated annealing technique practical, we need to find a good initial guess to be used as a starting point for this algorithm. In the following, we describe a very fast heuristic method.

The probability of missing the cancer can be expressed as a product of conditional probabilities,

$$
\begin{aligned}
P\left(B\left(x_{i}\right)=\mathrm{NC}, i=1, \ldots, K\right)= & P\left(B\left(x_{1}\right)=\mathrm{NC}\right) \bullet P\left(B\left(x_{2}\right)=\mathrm{NC} \mid B\left(x_{1}\right)=\mathrm{NC}\right) \bullet \\
& \ldots \bullet P\left(B\left(x_{K}\right)=\mathrm{NC} \mid B\left(x_{i}\right)=\mathrm{NC}, i=1, \ldots, K-1\right) .
\end{aligned}
$$

Our heuristic method sequentially minimizes a series of the conditional probabilities of missing cancer, each of them being one of the terms in (2). Suppose that there are $N$ different locations in the prostate template, and $M$ prostate samples in the training set. To minimize (2), one of $K$ biopsies, let's say the first biopsy $B\left(x_{1}\right)$, will be taken from the location $x_{1}$ where the likelihood of cancer is the highest, that is, the first term in (2), $P\left(B\left(x_{1}\right)=\mathrm{NC}\right)$, is the lowest. Knowing the location of the first biopsy $x_{1}$, in order to calculate the conditional probability $P\left(B\left(x_{2}\right)=\mathrm{NC} \mid B\left(x_{1}\right)=\mathrm{NC}\right)$, we remove those prostate samples that have cancer at location $x_{1}$, since those do not satisfy the condition $B\left(x_{1}\right)=\mathrm{NC}$, and recalculate the probability at each location. It is important to note that if the incidence of cancer at a location other than $x_{1}$ is strongly related with the incidence of cancer at location $x_{1}$, then the cancer occurrence probability of this location will become very low in the conditional probability $P\left(B\left(x_{2}\right)=\mathrm{NC} \mid B\left(x_{1}\right)=\mathrm{NC}\right)$. This is because all subjects with cancer at $x_{1}$ have been excluded in calculating the conditional probability $P\left(B\left(x_{2}\right)=\mathrm{NC} \mid B\left(x_{1}\right)=\mathrm{NC}\right)$. This implies that the second biopsy will most likely not be placed at the cancer locations strongly related with the location $x_{1}$. With this new conditional probability, the biopsy location $x_{2}$ can be determined by selecting the location where the likelihood of cancer is highest, i.e. the conditional 
probability $P\left(B\left(x_{2}\right)=\mathrm{NC} \mid B\left(x_{1}\right)=\mathrm{NC}\right)$ is lowest. Using the same procedure, the locations of other biopsy sites can be similarly determined. Effectively, this procedure minimizes each of the terms in (2) sequentially, rather than operating at the full joint distribution, and is therefore extremely fast.

\subsection{Increasing Robustness of Our Biopsy Strategy}

In order to make our biopsy strategy robust to the errors from needle placement, each cancer probability (or conditional cancer probability) function is spatially smoothed prior to finding optimal needle locations. This is specifically designed to avoid the cases that optimal needle locations are determined to be on isolated, high-probability regions, since those regions might be difficult to accurately sample in practice due to unavoidable errors in placing needles. With this formulation, our biopsy strategy is robust, since we guarantee that the optimal needle locations be on the regions with both high cancer probability and relatively wider spatial extent. Therefore, even a needle is wrongly placed in the neighborhood around the expected location, we still have very higher probability in detecting cancer.

\section{Experiments}

Two groups of experiments are performed in this section, in order to 1) test the performance of our deformable registration, 2) validate the predictive power of our atlasbased optimal biopsy strategies in detecting prostate cancer. These two experiments were performed on 64 of the 281 prostate subjects in our database.

\subsection{Performance on AFDM-Based Deformable Registration}

Fig. 4 demonstrates a procedure of registering and warping a representative prostate subject (Fig. 4(b1)) to the space of the prostate template (Fig. 4(a1)). In both of Figs. 4(a1) and 4(b1), the orange surfaces denote the prostate capsule, and the yellow surfaces denote the urethra. The red region in the subject denotes the positions of cancer. The side views of the template and the subject are shown in Fig. 3. The spatially normalized version of the subject is shown in Fig. 4(c1), where the white mesh corresponds to the capsule of the template and the red mesh corresponds to the capsule of the subject. Fig. 4(a2) shows a typical cross-sectional image of the template prostate. The corresponding cross-sectional image of the subject's prostate is shown in Fig. 4(b2). Notable are the shape differences between these two cross-sections. After using the warping algorithm, we obtain the warped image of Fig. 4(c2), whose shape is very similar to that of the template prostate in Fig. 4(a2).

We also measured the registration accuracy of AFDM. We did this by measuring the percent overlap and average distance between the prostate structures in the 64 images and their counterparts in the prostate template, after all prostate subjects had spatially normalized to the template. Note that these images are all labeled, and therefore overlap of the various prostate structures across subjects can be readily computed. For 64 prostate samples, their percent overlap measures ranged from $96.4 \%$ to $98.4 \%$, with the mean overlap $97.7 \%$. Their average boundary distances ranged from 
1.7 to 3.0 pixels, which is very small compared to the prostate image with the size of 256x256x124 pixels.

\subsection{Testing Predictability of the Statistical Atlas}

Using the registration and warping algorithm, we can eliminate the overall shape differences across individuals. In this way, we can find the spatial distribution of cancer within the space of the prostate template, which can be used to determine the needle biopsy strategy. We tested the heuristic sequential optimization procedure on 64 subjects $(M=64)$. All $N=256 \times 256 \times 124$ voxels were considered to be candidate biopsy locations. In Fig. 5(a), the optimal biopsy sites are shown as small red/yellow spheres and the underlying spatial statistical distribution of cancer is shown as green. Brighter green indicates higher likelihood for finding cancer in that location. The viewing angle of the statistical distribution is similar in both of Figs. 5(a) and 5(b), to facilitate comparison. In Fig. 5(b), the prostate capsule is shown as red. A typical cross-section of the 3D cancer distribution is also shown in Fig. 5(d). Six needles were adequate to detect the tumor $100 \%$, in those 64 subjects. The locations of 6 needles with depth information are shown in Fig. 5(c). Of course, this will likely not be the case as we increase the number of subjects. But, an important implication is that optimized needle placement is not necessarily on regions that have high likelihood of cancer. As we can see from Fig. 5(a), only first three yellow needles were placed in brighter green (high likelihood) regions. The rest three were placed in regions that were almost statistically independent from the first three.

We validated the predictive power of our statistical atlas using the leave-one-out method. For each time, we selected one subject from our 64 prostate samples, and we regenerated the statistical atlas by leaving this subject out. We then determined again the optimal biopsy sites, and we applied them to this left-out subject. This way, we measured the probability of missing the cancer. For 6-biopsy strategy, the leave-oneout method showed that the rate of success was $96.9 \%$.

In Fig. 6, we also provide the rates of success, as a function of the number of the needles $(K)$ and the volume of tissue that the needle actually extracts from the patient. Suppose that the volume extracted by a needle is $V$. In the reality there are errors in placing a needle, or in determining angle. Accordingly, we assume that the needle will actually extract only a part of the tissue thought to be extracted under ideal conditions in the optimized $K$-biopsy. If each needle actually extracts $60 \% \sim 100 \%$ of $V$ around its optimized location, then identical curves of success rates are obtained, as shown as the curve with 'o'. 6 needles are adequate to detect the tumor $100 \%$, while five needles can detect the tumor in 63 out of 64 cases. For other percentages of actual vs. theoretical volume extraction, i.e. $50 \%, 30 \%, 10 \%$, the rates of success with the number of the needles are shown as curves with ' $\checkmark,{ }^{\prime}$ ', ' $\boldsymbol{\square}$ '. For all these cases, 7 or 8 needles are adequate to detect the tumor $100 \%$. This numerical figure of success rate is very valuable for clinicians using our method, since they will be able to quantitatively evaluate the trade-off between success rate and patient discomfort. 


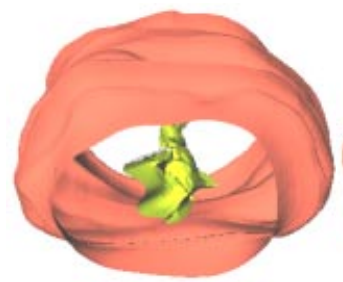

(a1)

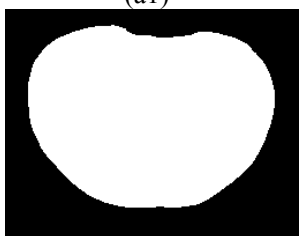

(a2)

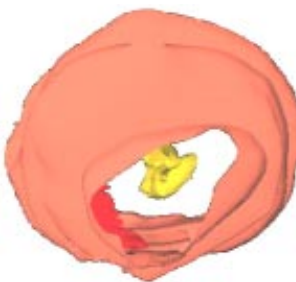

(b1)

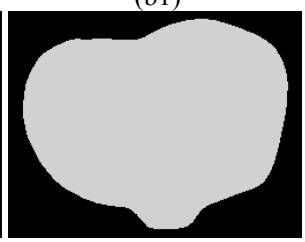

(b2)

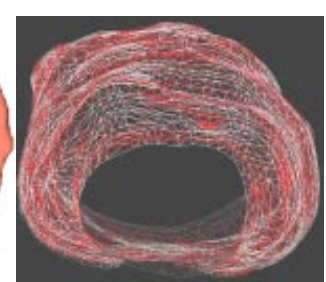

(c1)

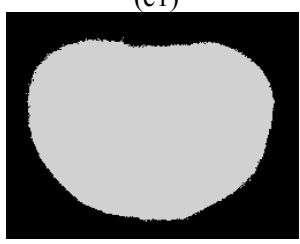

(c2)

Fig. 4. Results of the deformable registration technique in registering prostates. (a1) A prostate template to which all subjects are warped, (b1) a selected subject, (c1) overlay of the prostate boundary of the subject of b1 (red) after deformable registration with the template prostate (white). Figs. (a2-c2) are representative cross-sectional images corresponding to (a1-c1).
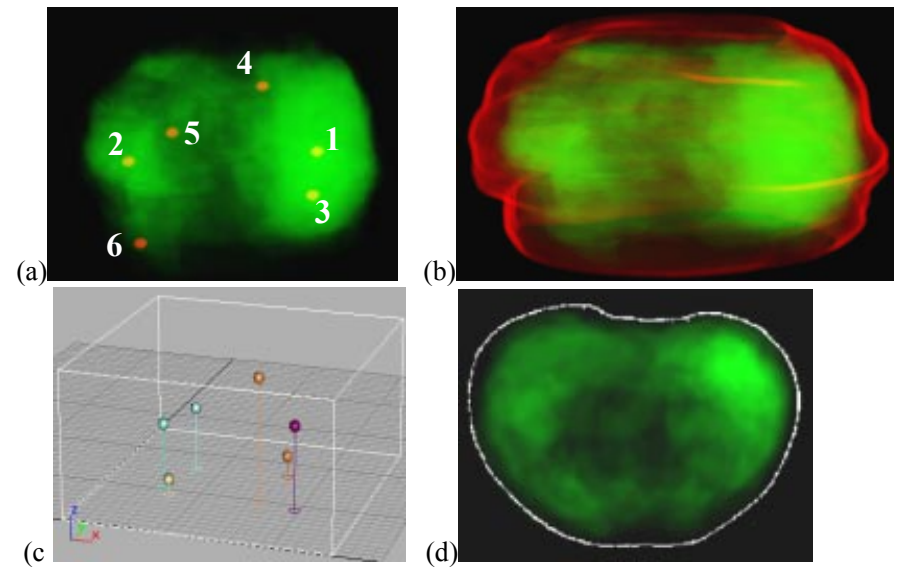

(b)

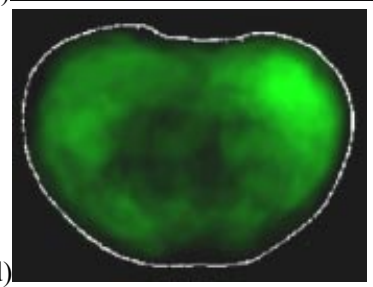

Fig. 5. Optimal biopsy strategy using statistical atlas of cancer distribution. The 6 biopsy positions are shown as small color spheres in $(\mathrm{a}, \mathrm{c})$, with the statistical atlas of cancer shown as green in $(a, b, d)$. Prostate capsule is shown as red in (b) for comparison. The cross-sectional image of the statistical atlas of cancer is shown in (d), where prostate capsule is shown as white. 


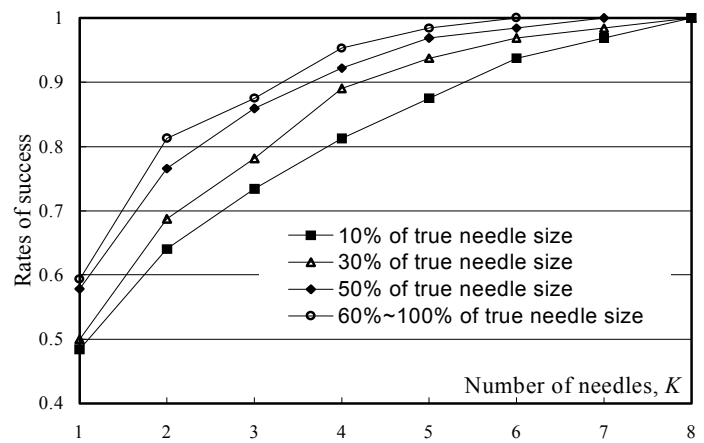

Fig. 6. The rates of success changing with the number of needles $K$ and the percentages of volume of tissue that the needle actually extracts from the expected neighbor of the optimized needle location.

\section{Summary and Conclusion}

We have presented a methodology to create a statistical atlas for designing optimal needle biopsy strategies. We have applied this method on 64 subjects. We will finally test this method on the whole database of 281 prostate subjects. We will also study the method of warping the statistical atlas to the ultrasound images of the patients' prostate, for image-guided biopsy and therapy.

Acknowledgments: We thankfully acknowledge the collaborations of John Bauer (Walter Reed Army Medical Center), Wei Zhang and Isabell Sesterhenn (Armed Forces Institute of Pathology), Judd Moul (Center for Prostate Disease Research), and Seong K. Mun (Georgetown University Medical Center). This work was supported in part by a grant from the National Science Foundation to the Center for Computer Integrated Surgical Systems and Technology. J. Zeng was supported by the Whitaker Foundation.

\section{References}

1. K.K. Hodge , J.E Mcneal, M.K. Terris, T.A. Stamey. Random systematic versus directed ultrasound guided transrectal core biopsies of the prostate. J. Urol., 142: 71-74, 1989.

2. R.C. Flanigan, W.J. Catalona, J.P. Richie, et al. Accuracy of digital rectal examination and transrectal ultrasonography in localizing prostate cancer. J. Urol., 152: 1506-1509, 1994.

3. A.L. Eskew, R.L. Bare, D.L. McCullough. Systematic 5-region prostate biopsy is superior to sextant method for diagnosing carcinoma of the prostate. J. Urol., 157: 199-202, 1997.

4. M.E. Chen, P. Troncoso, D.A. Johnston, K. Tang, R.J. Babaian. Optimization of prostate biopsy strategy using computer based analysis. J. Urol., 158(6): 2168-2175, Dec 1997.

5. J. Zeng, J.J. Bauer, A. Sofer, et al. Distribution of prostate cancer for optimized biopsy protocols. MICCAI, Pittsburgh, Oct. 2000.

6. D. Shen, C. Davatzikos. An adaptive-focus deformable model using statistical and geometric information. IEEE Trans. on PAMI, 22(8):906-913, August 2000.

7. D. Shen, E. Herskovits, and C. Davatzikos. An adaptive-focus statistical shape model for segmentation and shape modeling of 3D brain structures. IEEE Trans. on Medical Imaging, 20(4):257-270, April 2001.

8. C. Davatzikos. Spatial transformation and registration of brain images using elastically deformable models. Comp. Vis. and Image Understanding, 66(2):207-222, May 1997. 